\title{
Heparin-binding EGF-like growth factor and enteric neural stem cell transplantation in the prevention of experimental necrotizing enterocolitis in mice
}

\author{
Jia Wei', Yu Zhou' and Gail E. Besner ${ }^{1}$
}

BACKGROUND: Necrotizing enterocolitis (NEC) is associated with loss of neurons and glial cells in the enteric nervous system (ENS). Our goal was to determine whether enteric neural stem cell (NSC) transplantation, in conjunction with heparin-binding epidermal growth factor-like growth factor (HB-EGF), could protect against experimental NEC.

METHODS: In vitro, HB-EGF on NSC proliferation and migration, and the effects of receptors utilized by HB-EGF to exert these effects, were determined. In vivo, mouse pups were exposed to experimental NEC and treated with NSC alone, HB-EGF alone, NSC+HB-EGF, or HB-EGF overexpressing NSC. NSC engraftment and differentiation into neurons in the ENS, intestinal injury, intestinal permeability, and intestinal motility were determined.

RESULTS: HB-EGF promoted NSC proliferation via ErbB-1 receptors and enhanced NSC migration via ErbB-1, ErbB-4, and Nardilysin receptors. HB-EGF significantly enhanced the engraftment of transplanted NSC into the ENS during NEC. NSC transplantation significantly reduced NEC incidence and improved gut barrier function and intestinal motility, and these effects were augmented by simultaneous administration of HB-EGF or by transplantation of HB-EGF overexpressing NSC.

CONCLUSION: HB-EGF promotes NSC proliferation and migration. HB-EGF and NSC reduce intestinal injury and improve gut barrier function and intestinal motility in experimental NEC. Combined HB-EGF and NSC transplantation may represent a potential future therapy to prevent NEC.

$\mathbf{N}$ ecrotizing enterocolitis (NEC) is the most common cause of gastrointestinal mortality in newborns, typically affecting babies born prematurely with low birth weight. NEC affects $\sim 7 \%$ of infants with a birth weight between 500 and $1,500 \mathrm{~g}$. The overall mortality rate of NEC is $20-30 \%$ (ref. 1). Despite intensive research, the incidence and mortality of NEC have remained unchanged over the past six decades.

The enteric nervous system (ENS) is a collection of neurons and supportive glial cells organized in ganglia throughout the gastrointestinal tract (2). The ENS controls intestinal motility, modulates visceral sensation, and regulates intestinal blood supply and secretion of digestive hormones (3). In addition, the ENS modulates the proliferation and differentiation of intestinal epithelial cells via the secretion of distinct neuromediators (4). ENS immaturity in premature babies leads to poor intestinal motility and resultant bacterial overgrowth and translocation, which may increase vulnerability to NEC (5). We and others have documented ENS abnormalities in the intestines of patients with NEC, suggesting that an immature ENS may predispose premature infants to develop the disease (6). Furthermore, we have shown that the ENS injury associated with acute clinical NEC persists for months, suggesting that the ENS is restricted in its ability to recover from injury (6).

Stem cell therapy holds a powerful potential to treat ENS abnormalities by replacing damaged neurons with transplanted cells. Enteric neural stem cells (NSC) may represent an ideal source of stem cells for neurotransplantation since these cells are committed to differentiate into neurons and glial cells after transplantation. It has been reported that transplanted NSC isolated from human neonates can colonize aganglionic gut and differentiate into neurons and glial cells (7). However, NSC transplantation has limited success due to the low survival rate of the engrafted cells after transplantation (8).

Heparin-binding epidermal growth factor-like growth factor (HB-EGF) was initially isolated from cultured human macrophages (9), and later identified as a member of the epidermal growth factor (EGF) family (10). HB-EGF is a potent mitogen and chemotactic factor, and we have previously demonstrated that enteral administration of HB-EGF protects the intestines from histologic injury (11), promotes enterocyte migration and proliferation (12), and preserves intestinal stem cell viability during experimental NEC (13). We also showed that administration of HB-EGF enhances mesenchymal stem cell engraftment into injured intestine and promotes the efficacy of mesenchymal stem cell transplantation in animal models of NEC (14) and intestinal ischemia/reperfusion injury (15). We therefore hypothesized that HB-EGF may enhance the effect of NSC transplantation in NEC and augment the therapeutic 
effects of NSC transplantation. Our current goal was to investigate whether NSC transplantation would protect the ENS and intestine from NEC, and whether administration of HB-EGF in conjunction with NSC, or overexpression of HB-EGF in NSC, would lead to enhanced efficacy.

\section{RESULTS}

\section{HB-EGF Increases NSC Viability and Promotes NSC Migration} In Vitro

HB-EGF increased the number of viable NSC in a dosedependent manner, with addition of HB-EGF at 50, 75, or $100 \mathrm{ng} / \mathrm{ml}$ leading to significant increases in the number of viable NSC (Figure 1a). HB-EGF also promoted NSC migration in a dose-dependent manner, with addition of HB-EGF at 25,50 , or $75 \mathrm{ng} / \mathrm{ml}$ leading to significant increases in NSC migration (Figure 1b).

\section{HB-EGF Promotes NSC Viability via ErbB-1 and Promotes NSC Migration via ErbB-1, ErbB-4, and Nardilysin}

To determine which receptors are utilized by HB-EGF in promoting NEC viability and migration, we first used RT-PCR to determine the expression of ErbB-1, ErbB-2, ErbB-3, ErbB-4, and Nardilysin in NSC and found that NSC express all of these receptors (Figure 2a). We then transfected NSC with siRNA to each individual receptor to decrease expression of the receptor. Quantitative real-time PCR confirmed significantly decreased expression of ErbB-1, ErbB-2, ErbB-3, ErbB-4, and Nardilysin mRNA after respective siRNA transfection (Figure 2b). Transfection with siRNA to ErbB-1 significantly decreased HB-EGF-mediated NSC viability compared with transfection with scrambled siRNA, whereas HB-EGF-mediated NSC viability was not affected by transfection of NSC with siRNA to ErbB-2, ErbB-3, ErbB-4, or Nardilysin, suggesting that the effects of HB-EGF on NSC viability are mediated solely via ErbB-1 (Figure 2c). We next investigated the effect of these receptors on NSC migration. We found that transfection of NSC with siRNA to ErbB-1, ErbB-4, and Nardilysin significantly decreased HB-EGF-mediated NSC migration compared with scramble siRNA transfection, whereas HB-EGF-mediated NSC migration was not affected by transfection of NSC with siRNA to ErbB-2 or ErbB-3 (Figure 2d). These results suggest that the effects of HB-EGF on NSC migration are mediated via ErbB-1, ErbB-4, and Nardilysin.

\section{Enterally Administered HB-EGF Increases NSC Proliferation In Vivo}

To determine whether HB-EGF promotes the proliferation of engrafted enhanced green fluorescent protein (EGFP)-labeled NSC in vivo, we performed double immunohistochemical staining for EGFP and Ki67 (proliferation cell marker) in intestinal sections from pups exposed to NEC+NSC or to NEC+NSC+HB-EGF. We found that the percentage of cells that express both Ki67 and GFP/total GFP-positive cells was significantly increased in the NEC+NSC+HB-EGF group compared with the NEC+NSC group, indicating that enterally administered HB-EGF increases NSC proliferation in vivo (Figure 3a). a

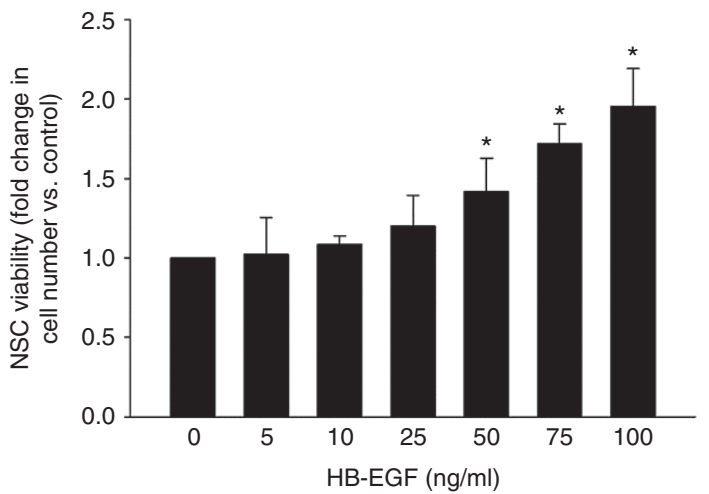

b

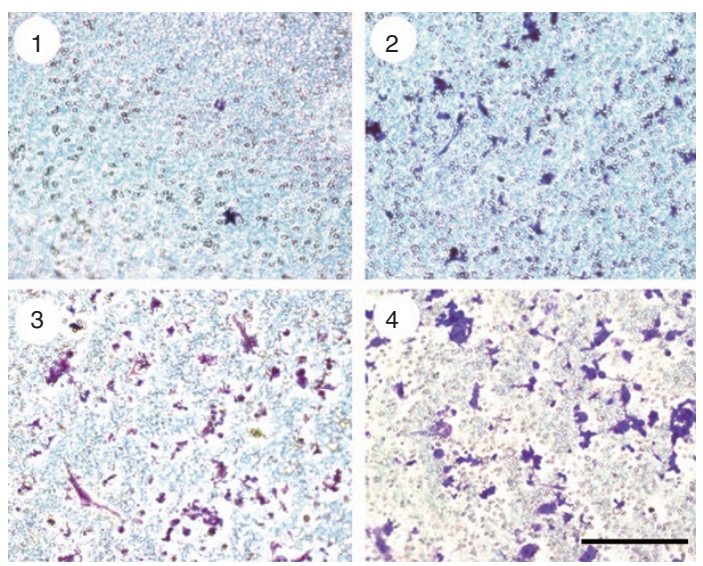

C

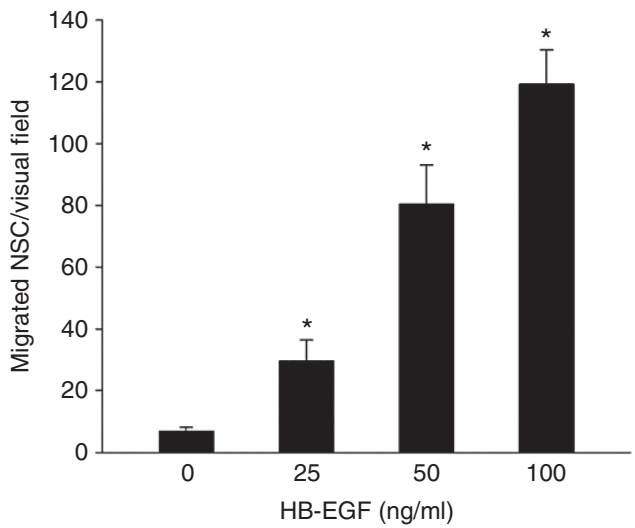

Figure 1. HB-EGF promotes NSC viability and migration. (a) Effect of HB-EGF on NSC viability. ${ }^{*} P<0.05$ vs. no addition. (b) Representative crystal violet stained images of migrated NSC trapped in polycarbonate membrane inserts in the presence of (i) no HB-EGF; (ii) HB-EGF $25 \mathrm{ng} /$ $\mathrm{ml}$; (iii) HB-EGF $50 \mathrm{ng} / \mathrm{ml}$; (iv) HB-EGF $100 \mathrm{ng} / \mathrm{ml}$, with quantification of NSC migration shown in the bar graph. Scale bar $=50 \mu \mathrm{m}$. Magnification: $\times 100 .{ }^{*} P<0.05$ vs. no addition. All values represent mean \pm SD. HB-EGF, heparin-binding epidermal growth factor-like growth factor; NSC, neural stem cell.

HB-EGF Enhances the Engraftment of Transplanted NSC Into the ENS During NEC

We next tested the effects of HB-EGF on EGFP-labeled NSC in our experimental NEC model in vivo. We first confirmed that transfection of murine NSC with a human HB-EGF expression plasmid led to significantly increased expression of human HB-EGF mRNA in HB-EGF-transfected 
a
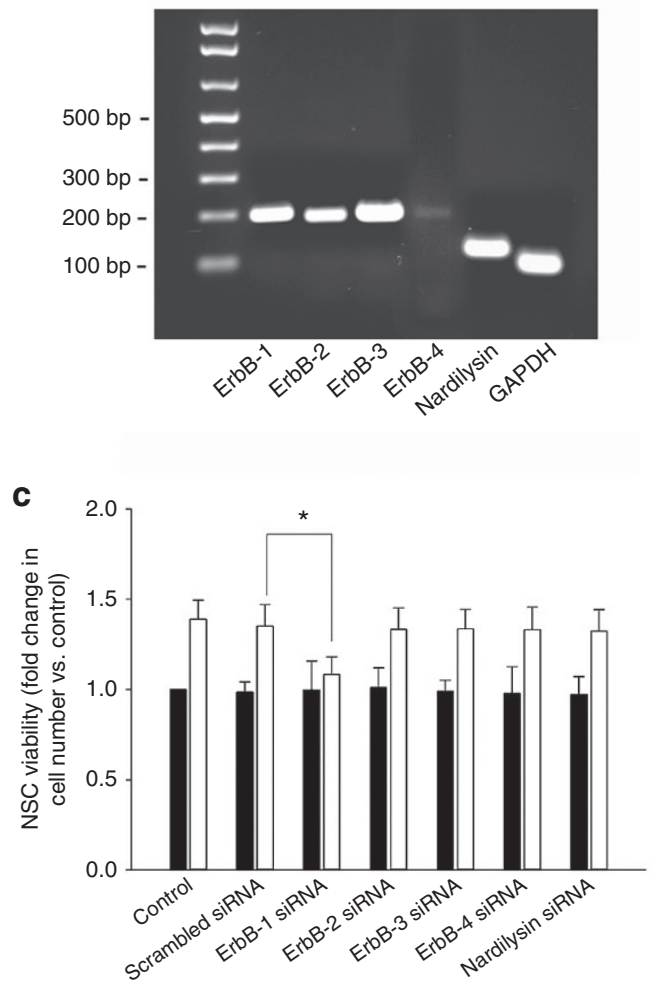
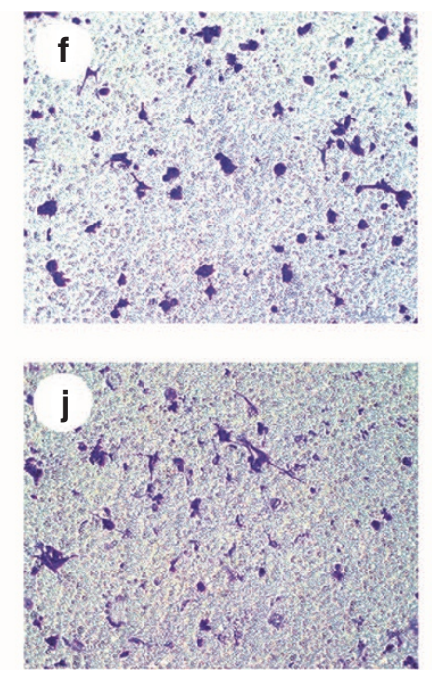

b

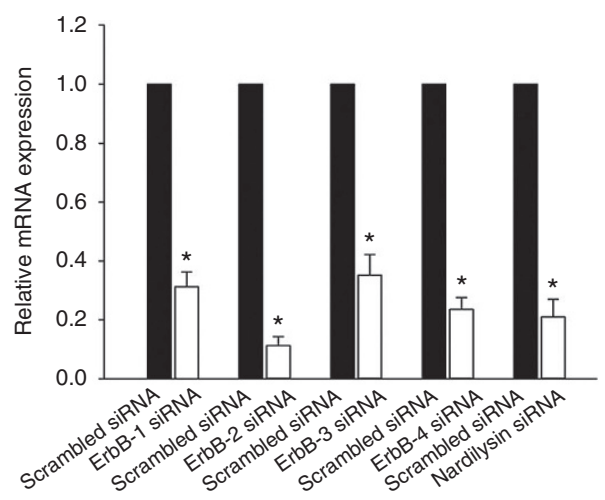

d

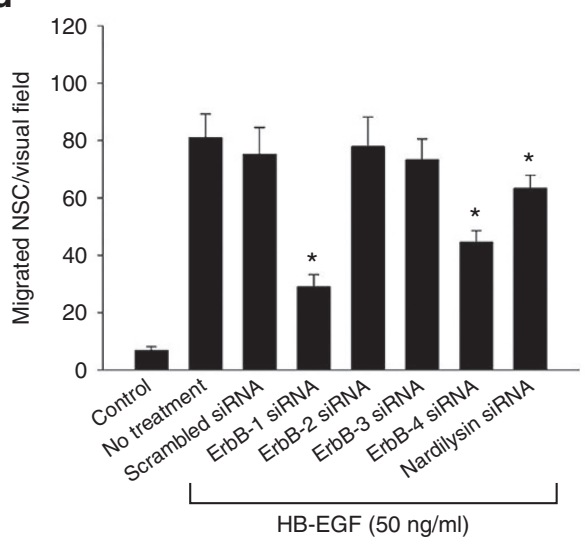

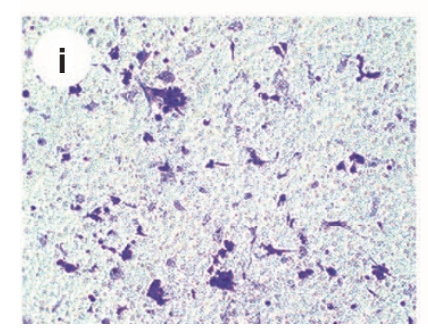

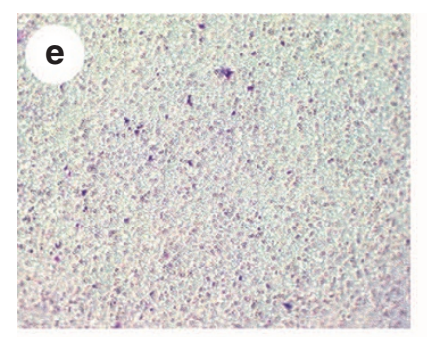

Figure 2. HB-EGF promotes NSC viability via ErbB-1 and enhances NSC migration via ErbB-1, ErbB-4, and Nardilysin. (a) RT-PCR of ErbB and Nardilysin receptor mRNA expression in NSC. (b) Silencing efficiency of indicated siRNAs in NSC as determined by real-time PCR. ${ }^{*} P<0.05$ vs. scrambled siRNA. (c) Effect of receptor silencing on NSC viability. Black bars, control; white bars, HB-EGF. ${ }^{*} P=0.02$. (d) Effect of receptor silencing on HB-EGF-mediated NSC migration. ${ }^{*} P<0.01$ vs. scramble siRNA-transfected NSC+HB-EGF $(50 \mathrm{ng} / \mathrm{ml})$. The images represent migration of: (e) NSC with no addition; (f) NSC+ HB-EGF ( $50 \mathrm{ng} / \mathrm{ml}) ;(\mathbf{g})$ scramble siRNA-transfected NSC+HB-EGF $(50 \mathrm{ng} / \mathrm{ml})$; (h) ErbB-1 siRNA-transfected NSC+HB-EGF (50 ng/ml); (i) ErbB-2 siRNA transfected NSC+HB-EGF (50 ng/ml); (j) ErbB-3 siRNA-transfected NSC+HB-EGF (50 ng/ml); (k) ErbB-4 siRNA-transfected NSC+HB-EGF (50 ng/ml); (l) Nardilysin siRNA-transfected NSC+HB-EGF $(50 \mathrm{ng} / \mathrm{ml})$. Scale bar $=50 \mu \mathrm{m}$. Magnification: $\times 100$. All values represent mean \pm SD. HB-EGF, heparin-binding epidermal growth factor-like growth factor; NSC, neural stem cell.

NSC compared with scramble-transfected NSC. Double immunofluorescence staining for EGFP and human HB-EGF was performed in intestinal sections from pups exposed to NEC + scramble transfected NSC or NEC+HB-EGF-overexpressing NSC. We confirmed that
HB-EGF-overexpressing NSC produced easily detectable HB-EGF protein in vivo (Figure 3b). Double immunofluorescence staining of intestinal sections was also performed for EGFP and for the pan neuronal cell marker $\mathrm{HuC} / \mathrm{D}$ (Figure 3c). Engrafted EGFP-positive cells were distributed 
a

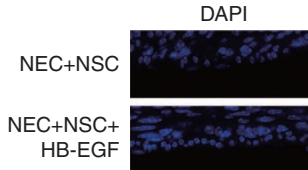

b

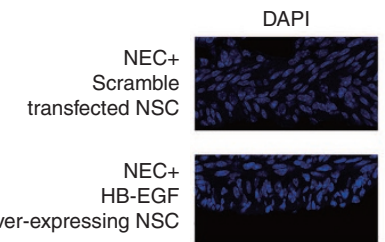

C
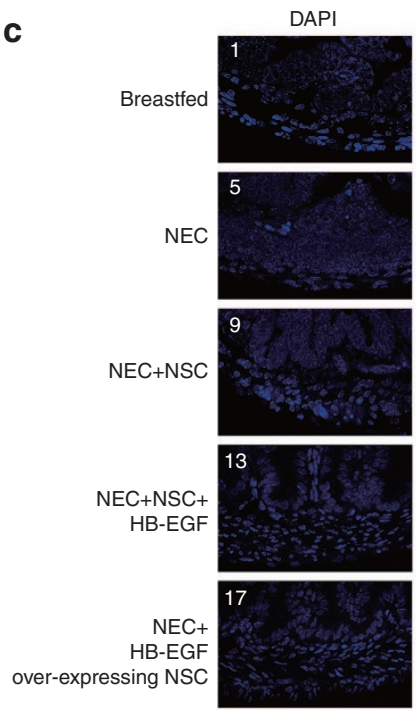

d

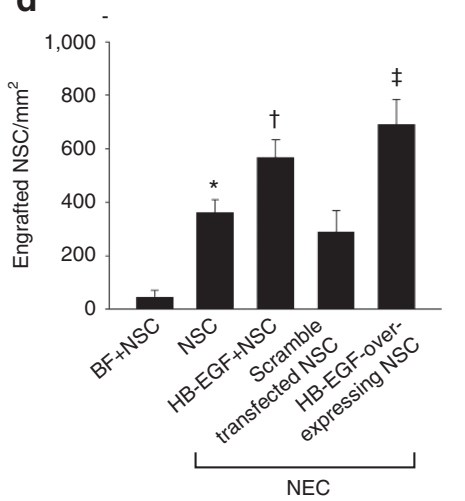

GFP

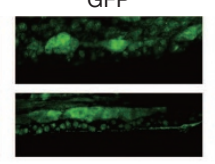

EGFP
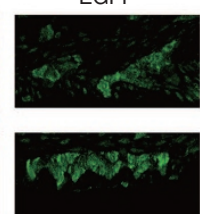

EGFP
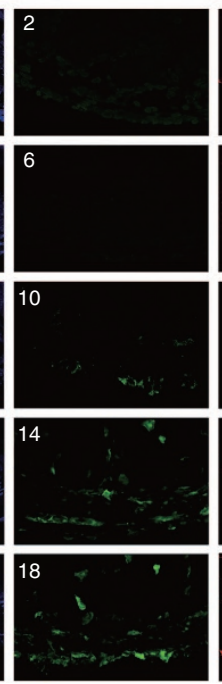

e

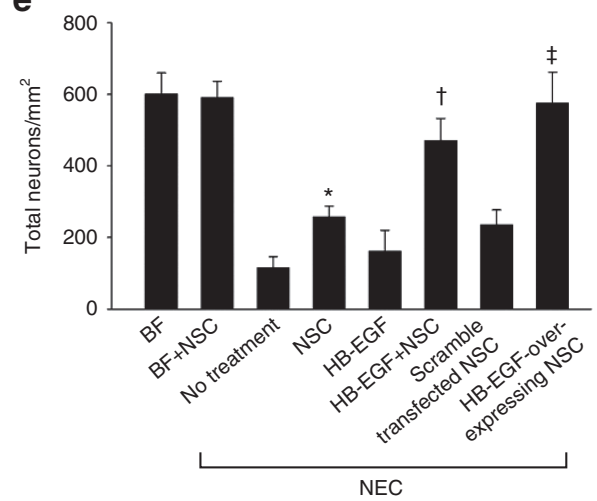

Human-HB-EGF
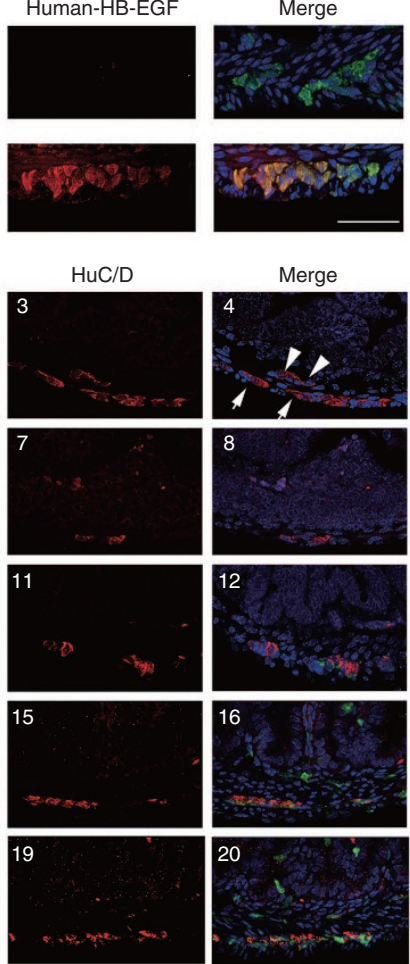

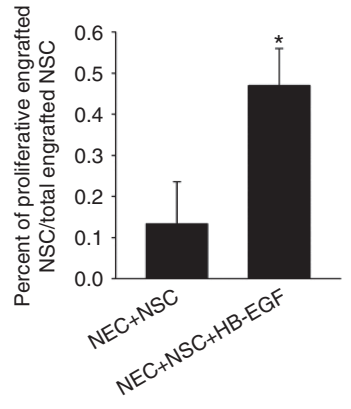

f

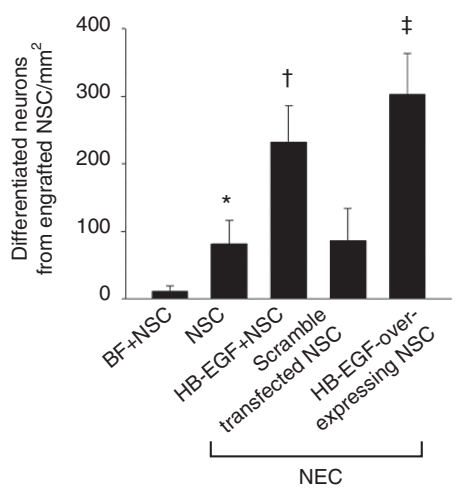

Figure 3. Effect of HB-EGF on neural stem cell engraftment, proliferation, and differentiation in intestines of pups subjected to experimental NEC.

(a) Engrafted NSC proliferation. Images of engrafted NSC from pups exposed to NEC+NSC or NEC+NSC+HB-EGF are shown. Cell nuclei are indicated by DAPI staining, engrafted NSCs are indicated by EGFP staining (green), and proliferative cells are indicated by Ki67 staining (red). Scale bar $=50 \mu \mathrm{m} ;$ Magnification: $\times 40$. Quantification of proliferative engrafted NSC/total engrafted NSC is shown in the bar graph. ${ }^{*} P<0.05$ vs. NEC+NSC. (b) Production of human HB-EGF protein by engrafted NSC. Images of engrafted NSC from pups exposed to the NEC+scramble-transfected NSC or NEC+HB-EGF-overexpressing NSC are shown. Cell nuclei are indicated by DAPI staining, engrafted NSCs are indicated by EGFP staining (green), and human HB-EGF protein is indicated by HB-EGF staining (red). Scale bar $=50 \mu \mathrm{m}$. Magnification: $\times 40$. (c) Images of the ENS from pups exposed to the following treatments (1-4): breast feeding; (5-8) NEC; (9-12): NEC+NSC i.p.; (13-16): NEC+enteral HB-EGF+NSC i.p.; (17-20): NEC+HB-EGF-overexpressing NSC i.p.. Cell nuclei are indicated by DAPI staining, engrafted NSCs are indicated by EGFP staining (green), and neurons are indicated by HuC/D staining (red). Some engrafted NSCs have differentiated into mature neurons as demonstrated by colocalization of both EGFP and HuC/D staining. White arrowheads, submucosal plexus; white arrows, myenteric plexus. Scale bar $=50 \mu \mathrm{m}$. Magnification: $\times 40$. (d) Quantification of engrafted NSC. ${ }^{*} P<0.05$ vs. BF+NSC; ${ }^{\dagger} P<0.05$ vs. NEC+NSC; ${ }^{\ddagger} P<0.05$ vs. NEC+NSC and vs. NEC+scramble-transfected NSC. (e) Quantification of total neurons. ${ }^{*} P<0.05$ vs. nontreated NEC; ${ }^{+} P<0.05$ vs. nontreated NEC and vs. NEC + NSC and vs. NEC $+\mathrm{HB}-\mathrm{EGF} ;{ }^{\ddagger} P<0.05$ vs.

$\mathrm{NEC}+\mathrm{NSC}$ and vs. NEC+scramble transfected NSC. (f) Quantification of differentiated neurons from engrafted NSC $* P<0.05 \mathrm{vs}$. BF+NSC; ${ }^{+} P<0.05$ vs. NEC+NSC; ${ }^{\ddagger} P<0.05$ vs. NEC+NSC and vs. NEC+HB-EGF+NSC and vs. NEC+scramble-transfected NSC. All values represent mean \pm SD. HB-EGF, heparin-binding epidermal growth factor-like growth factor; NEC, necrotizing enterocolitis; NSC, neural stem cell. 
in the muscularis and mucosa of the recipient intestine, including the submucosal and myenteric plexuses. A subset of EGFP-positive cells were immunoreactive to $\mathrm{HuC} / \mathrm{D}$, indicating transplanted NSC that differentiated into mature neurons. Pups exposed to experimental NEC that received NSC i.p. had significantly increased NSC engraftment into the intestines compared with breastfed pups that received NSC i.p., demonstrating that NSC preferentially engraft into NEC-injured intestine rather than intact intestine (Figure 3d). In pups subjected to NEC, administration of enteral HB-EGF in conjunction with NSC transplantation led to significantly increased NSC engraftment compared with administration of NSC alone. HB-EGF-overexpressing NSC demonstrated similar effects, with increased engraftment into NEC-affected intestine compared with administration of either nontransfected NSC or scramble-transfected NSC.

\section{HB-EGF Promotes Engrafted NSC Differentiation and Protects the ENS From NEC Injury}

Significant enteric neuronal loss was found in pups exposed to NEC compared with pups that were breast-fed, confirming ENS injury during NEC (Figure 3e). Administration of enteral HB-EGF in conjunction with NSC transplantation led to a significant increase in total neurons in the intestine compared with pups subjected to NEC that were treated with NSC alone. Administration of HB-EGF-overexpressing NSC led to significantly increased neurons in the intestine compared with administration of either nontransfected NSC or scrambletransfected NSC. Lastly, we quantified differentiated neurons derived from engrafted NSC (cells double stained for EGFP and $\mathrm{HuC} / \mathrm{D}$ ). Administration of enteral HB-EGF in conjunction with nontransfected NSC led to a significant increase in the number of differentiated neurons derived from engrafted NSC compared with pups treated with nontransfected NSC alone (Figure 3f). Furthermore, administration of HB-EGFoverexpressing NSC led to a significant increase in the number of differentiated neurons derived from engrafted NSC compared with administration of either nontransfected NSC or scramble-transfected NSC. These results demonstrate that HB-EGF promotes NSC differentiation and protects the ENS from injury during NEC.

\section{HB-EGF and NSC Act Together to Reduce Intestinal Injury During Experimental NEC}

Representative images for each intestinal injury grade are shown in Figure 4a. Pups exposed to experimental NEC had a significantly increased incidence of histologic injury compared with breastfed pups (68.8 vs. $0 \% ; P<0.01$; Figure 4 b). Pups exposed to NEC that received NSC alone or enteral HB-EGF alone had a significantly decreased incidence of NEC compared with pups subjected to nontreated NEC ( 48.7 vs. $68.8 \%$; $P=0.047$ and 38.4 vs. $68.8 \% ; P=0.012$ ). A further decrease in the incidence of NEC was achieved with simultaneous administration of HB-EGF and NSC compared with treatment with NSC alone (25.8 vs. $48.7 \%$; $P=0.043)$. A significant decrease a
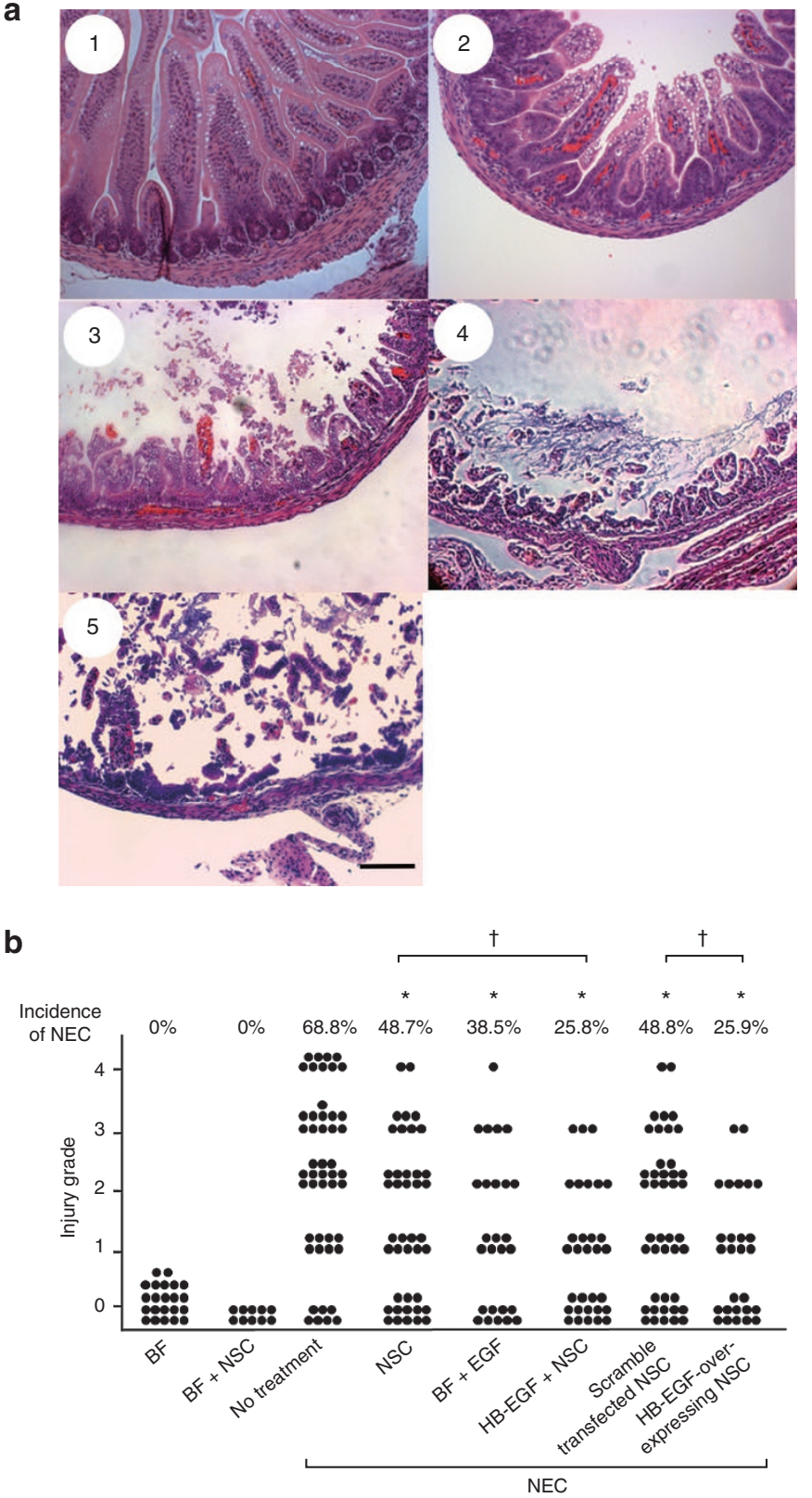

Figure 4. Effect of HB-EGF and neural stem cell transplantation on the incidence and severity of NEC. (a) Representative examples of histological scoring of intestines from mouse pups. Scale bar $=100 \mu \mathrm{m}$. Magnification: $\times 20$. (1) grade 0, normal intestine; (2) grade 1, epithelial cell lifting or separation; (3) grade 2, necrosis to mid villus level; (4) grade 3, necrosis of entire villus; (5) grade 4, transmural necrosis. Any injury of grade 2 or above is considered consistent with NEC. (b) Incidence and severity of NEC. Each dot represents an individual mouse pup. ${ }^{*} P<0.01$ vs. nontreated NEC; ${ }^{\dagger} P<0.05$. HB-EGF, heparin-binding epidermal growth factor-like growth factor; NEC, necrotizing enterocolitis.

in the incidence of NEC was also achieved by administration of HB-EGF-overexpressing NSC compared with scrambletransected NSC ( 25.9 vs. $48.8 \% ; P=0.048$ ). The efficacy of administration of HB-EGF-overexpressing NSC was equivalent to the efficacy of simultaneous administration of HB-EGF and NSC. 


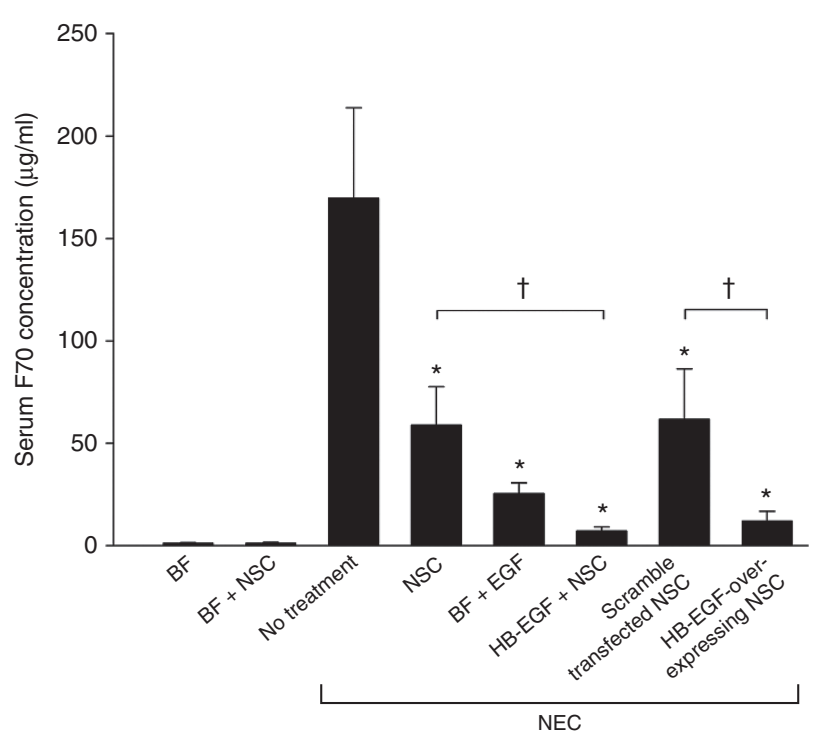

Figure 5. Effect of HB-EGF and neural stem cell transplantation on gut barrier function. Intestinal permeability was measured as the serum concentration of fluorescein isothiocyanante dextran that was administered enterally $4 \mathrm{~h}$ prior to euthanasia. Decreased serum FD70 values are indicative of improved gut barrier function. Values represent mean \pm SD. $n=12$ for each group. ${ }^{*} P<0.05$ vs. nontreated NEC. ${ }^{\dagger} P<0.05$. HB-EGF, heparin-binding epidermal growth factor-like growth factor; NEC, necrotizing enterocolitis.

HB-EGF and NSC Act Together to Preserve Gut Barrier Function Pups that were breastfed or breastfed with administration of NSC had intact gut barrier function with normal intestinal permeability (Figure 5a). Pups exposed to NEC had significantly increased intestinal permeability compared with pups that were breastfed, indicating gut barrier dysfunction associated with NEC. Pups exposed to NEC that received NSC alone or HB-EGF alone had significantly decreased intestinal permeability compared with pups subjected to nontreated NEC. A further significant decrease in intestinal permeability was achieved by simultaneous administration of HB-EGF and NSC compared with treatment with either NSC alone or with HB-EGF alone. Significantly decreased intestinal permeability was also achieved by administration of HB-EGFoverexpressing NSC compared with nontransfected NSC or scramble-transfected NSC. The efficacy of administration of HB-EGF-overexpressing NSC was equivalent to the efficacy of simultaneous administration of HB-EGF and NSC.

\section{HB-EGF and NSC Act Together to Promote Intestinal Motility}

Pups exposed to NEC had significantly decreased intestinal motility compared with breastfed pups as measured by methylene blue dye migration (Figure 6). There was increased intestinal motility in pups exposed to NEC that were treated with enteral HB-EGF or scramble transfected NSC compared with pups subjected to nontreated NEC. Pups exposed to NEC that received HB-EGF overexpressing NSC had intestinal motility that was completely restored to normal levels.
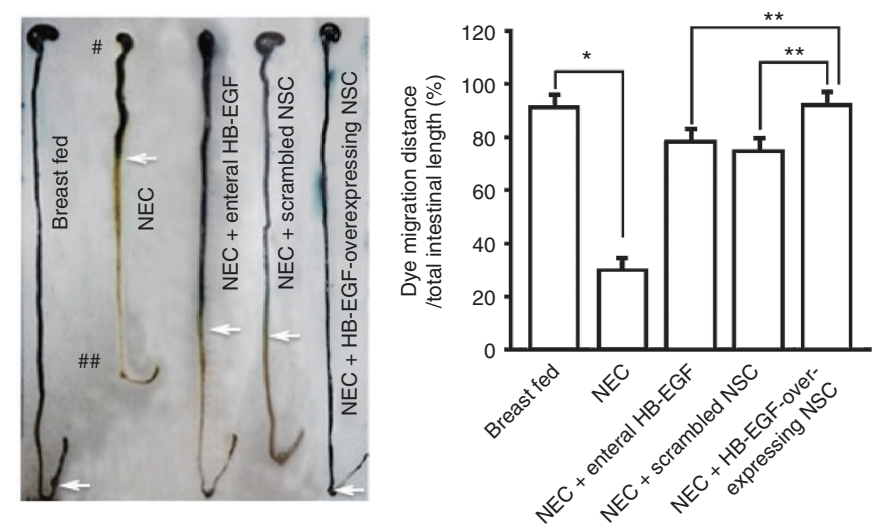

Figure 6. Effect of HB-EGF and neural stem cell transplantation on intestinal motility. Representative images of methylene blue dye migration. White arrows indicate the most distal point of dye migration. "stomach,

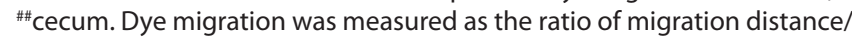
total intestinal length. Values represent mean \pm SD. $n=6$ for each group. ${ }^{*} P<0.01 ;{ }^{* *} P<0.05$. HB-EGF, heparin-binding epidermal growth factorlike growth factor; NEC, necrotizing enterocolitis.

\section{DISCUSSION}

Recent evidence suggests that an immature ENS may play a role in the pathogenesis of NEC. Furthermore, interventions to prevent ENS damage during NEC are highly desirable. In this study, we have shown that NSC transplantation and HB-EGF can protect the ENS and the intestines from NEC.

Enteric NSCs have characteristic self-renewing capabilities and terminally differentiate into neurons and glial cells, providing the potential to rebuild an impaired ENS. NSCs obtained from gut mucosal biopsy specimens in children undergoing endoscopy have been successfully used to generate neurospheres capable of proliferation and differentiation, and which generated ganglion-like structures including neurons and glial cells after transplantation into aganglionic human hindgut (16). The graft-derived neurons had morphological, neurochemical, and electrophysiological characteristics similar to enteric neurons (17). However, an inflammatory microenvironment can have significant negative impact on the survival, proliferation, and migration of grafted NSC (8). Proinflammatory cytokines such as IL-1 $\beta$ and IL- 6 suppress neuronal differentiation (18), potentially diminishing the beneficial effects of engrafted NSC. We have previously demonstrated that HB-EGF significantly reduces intestinal ischemia/ reperfusion injury-induced expression of the proinflammatory cytokines TNF- $\alpha$, IL-1 $\beta$, and IL-6 in vivo (19). We have also shown that HB-EGF protects many cell types including intestinal stem cells from injury (20) and promotes murine ENS development and enteric neural crest cell migration (21). In our current study, HB-EGF increased the number of viable NSC at least in part by increasing NSC proliferation, but may also act by decreasing NSC apoptosis. HB-EGF also promoted NSC migration. The combined effects of HB-EGF on inflammatory cytokines, NSC proliferaton, and NSC migration may enhance the therapeutic effects of NSC transplantation. 
Like other members of the EGF family, HB-EGF can interact with the four known EGF receptors (ErbB-1, ErbB-2, ErbB3, and ErbB-4). In addition, Nardilysin acts as an HB-EGFspecific receptor (22). The current study demonstrates that administration of HB-EGF promotes NSC proliferation via ErbB-1 and enhances NSC migration via ErbB-1, ErbB-4, and Nardilysin. HB-EGF/ErbB-4 signaling is known to be associated with proper development of neuromere/pharynegeal tissues during cranial neural crest cell migration to the periphery (23). Nardilysin is a highly specific receptor for HB-EGF (22), and high levels of Nardilysin transcripts are almost exclusively associated with neural tissues, indicating that Nardilysin may play an important role in neuronal development (24). Our results confirm that Nardilysin is involved in HB-EGFmediated NSC migration.

Our in vivo data show that administration of HB-EGF increases the numbers of transplanted NSC that engraft into the intestines during NEC, leading to decreased intestinal injury scores and improved gut barrier function. We also confirmed decreased intestinal motility in pups exposed to NEC and increased motility in pups exposed to NEC that were treated with enteral HB-EGF or with HB-EGF-overexpressing NSC. In addition, both HB-EGF and NSC transplantation led to increased numbers of neurons in the intestines of pups with NEC. This suggests that the therapeutic regimens employed can protect enteric neurons from NEC-induced injury and improve neuronal survival and function. Furthermore, some of the engrafted NSCs differentiate into mature neurons in the injured intestine.

To determine whether HB-EGF exerts its therapeutic effects in experimental NEC by acting directly on NSC, we transfected NSC with the HB-EGF gene to directly overexpress the growth factor in these cells. In this manner, we hoped to eliminate its beneficial effects on other cell types in the intestine. We found that transplantation of HB-EGF-overexpressing NSC had similar efficacy to administration of HB-EGF and nontransfected NSC in combination, suggesting that HB-EGF acts directly on NSC in our model by promoting proliferation or possibly by preserving NSC viability via decreased apoptosis and necrosis of the transplanted cells (20). Although it is impossible to completely rule out the possibility that HB-EGF protects the ENS from injury secondary to its effects in preserving mucosal integrity, previous work from our laboratory also showed that deletion of the HB-EGF gene directly leads to delayed migration of enteric neural crest cells and a dysfunctional ENS (21), indicating that HB-EGF has a direct effect on enteric neural crest cells and the ENS.

In summary, the results of our study show that HB-EGF promotes NSC viability via ErbB-1 receptors and enhances NSC migration via ErbB-1, ErbB-4, and Nardilysin receptors. HB-EGF enhances the engraftment of transplanted NSC into the ENS and promotes engrafted NSC proliferation and differentiation during experimental NEC in vivo. In addition, HB-EGF and NSC act together to reduce intestinal injury, to improve gut barrier function during NEC, and to restore intestinal motility upon recovery from NEC. Combined HB-EGF and NSC transplantation may represent a potential clinical therapy to prevent NEC in the future.

\section{METHODS}

\section{Animal Ethical Statement}

The following experimental protocol followed the guidelines for the ethical treatment of experimental animals as approved by the Institutional Animal Care and Use Committee of the Research Institute at Nationwide Children's Hospital (protocol \#02205 AR).

\section{NSC Culture}

NSCs were harvested from 11.5 day gestational age (E11.5) pan-EGFP mice (Jackson Laboratory, Bar Harbor, ME) and cultured as described (6). Fetal intestines were dissected and digested in Collagenase/ Dispase $(50 \mu \mathrm{g} / \mathrm{ml}$; Worthington Biochemical, Freehold, NJ) for $1 \mathrm{~h}$. Centrifuged tissue pellets were retrieved and cultured in Dulbecco's Modified Eagle Medium/Ham's Nutrient Mixture F12 (DMEM/ F12; Corning, Manassas, VA) supplemented 1:1 with $\mathrm{N}_{2}$ supplement (Gibco, Grand Island, NY), with the addition of mouse basic fibroblast growth factor (b-FGF, $20 \mathrm{ng} / \mathrm{ml}$; Sigma-Aldrich, St Louis, MO), mouse EGF (20 ng/ml; Sigma-Aldrich), 15\% chicken embryo extract (Gemini, West Sacramento, CA), and 1\% penicillin-streptomycin (Invitrogen, Carlsbad, CA). NSCs proliferated and grew as free-floating neurospheres, and the suspended neurospheres were collected by harvesting the culture medium. Neurospheres were confirmed to contain $>95 \%$ NSC as determined by staining with the stem cellspecific marker Nestin.

\section{NSC Transient Transfection}

HB-EGF plasmid transfection for overexpression of HB-EGF. Neurospheres were mechanically dissociated into single cells and transiently transfected with $4 \mu \mathrm{g}$ of pCMV6-Entry vector carrying full-length human HB-EGF plasmids (Origene, Rockville, MD) or with scrambled DNA control plasmid, using an Amaxa Basic Nucleofector kit (Lonza, Allendale, NJ) and the Amaxa Nucleofactor II apparatus (Lonza). Transfection efficiency was determined by real-time PCR using the following human HB-EGF primers: sense: $5^{\prime}$ - CTCTCCCTGCCAAGTCTCAG $-3^{\prime}$ and anti-sense: 5'-CTGCATGGAGTAGCACCAGA-3'. Transfected NSCs were incubated in DMEM/F12 medium supplemented with 5\% fetal bovine serum for $24 \mathrm{~h}$ prior to administration in vivo.

\section{Knockdown of ErbB and Nardilysin Receptor Expression}

Neurospheres were mechanically dissociated into single cells and transfected with $200 \mathrm{nmol} / \mathrm{l}$ of siRNA designed to knock down mouse ErbB-1, ErbB-2, ErbB-3, ErbB-4 or Nardilysin genes, or with scrambled siRNA (all from Ambion, Carlsbad, CA) using Amaxa Basic Nucleofector kit (Lonza) and the Amaxa Nucleofactor II apparatus (Lonza). siRNA suppression efficiency and plasmid transfection efficiency were determined by real-time PCR as follows. Total RNA from NSC was extracted using RNA STAT-60 (TEL-TEST, Friendswoods, TX) according to the manufacturer's protocol. Aliquots of $1 \mu \mathrm{g}$ of total RNA were reverse transcribed using SuperScript II Reverse Transcriptase (Invitrogen) and oligo-dT(18)-primers (Invitrogen). RT-PCR amplification was performed in a $25 \mu \mathrm{l}$ reaction containing $150 \mathrm{ng}$ cDNA, $12.5 \mu \mathrm{l} 2 \times$ QuantiTect PCR Master Mix (Qiagen, Valencia, CA), and $1 \mu \mathrm{l}$ of $10 \mathrm{nmol} / \mathrm{l}$ primers (Integrated DNA Technologies, Skokie, IL). The primers used are shown in Table 1. The real-time PCR was performed using the SYBR Green Master Mix kit and the ABI Prism 7000 Sequence Detection System (Applied Biosystems, Forster City, CA). Glyceraldehyde-3-phosphate dehydrogenase was amplified as an internal control.

\section{NSC Viability Assay}

NSCs were seeded as single cells into 96-well plates at a final concentration of 5,000 cells/well in culture medium containing b-FGF, chicken embryo extract, and $\mathrm{N}_{2}$ supplement. NSCs were cultured for $6 \mathrm{~d}$ in the presence of HB-EGF $(0-100 \mathrm{ng} / \mathrm{ml})$. Cell proliferation was determined by addition of the PrestoBlue Cell Viability Reagent (10 $\mu$; Life Technologies, Grand island, NY) for $30 \mathrm{~min}$, with 
Table 1. Primer sequences for amplification of mouse ErbB-1, ErbB-2, ErbB-3, ErbB-4, Nardilysin, or GAPDH

\begin{tabular}{|c|c|c|c|c|}
\hline Gene & Gene accession no. & Sense $\left(5^{\prime}-3^{\prime}\right)$ & Anti-sense $\left(5^{\prime}-3^{\prime}\right)$ & bp \\
\hline ErbB1 (mouse) & NM_207655.2 & GAAGCCACATCTCCAAAAGC & AGGAGGTACTGGGAGCCAAT & 200 \\
\hline ErbB2 (mouse) & NM_020507.3 & AGAGTCCCAACCACGTCAAG & AAGGTCATCAGCTCCCACAC & 193 \\
\hline ErbB3 (mouse) & NM_010153.1 & GGACAGTCCGGGAGATTACA & GACACGCCCAGCACTAATTT & 200 \\
\hline ErbB4 (mouse) & NM_010154.1 & TGCCATAAGTCTTGCACTGG & TGAAGTTCATGCAGGCAAAG & 200 \\
\hline Nardilysin (mouse) & NM_146150 & GGGGTCTGTGCGAAGAATCAT & CGCCCAAGTCCTGTCCATT & 131 \\
\hline GAPDH (mouse) & NM_008085 & AGCTTCGGCACATATTTCATCTG & CGTTCACTCCCATGACAAACA & 89 \\
\hline
\end{tabular}

GAPDH, glyceraldehyde-3-phosphate dehydrogenase.

quantification determined using a fluorescence reader (Molecular Devices, Sunnyvale, CA) with a 560/590 nm filter set. Each experiment was performed three times with each sample tested in triplicate.

\section{NSC Migration Assay}

NSC migration was assessed using transwell chambers with 8.0 $\mu \mathrm{m}$ pore polycarbonate membrane inserts (Greiner Bio-One, Frickenhausen, Germany) coated with laminin $(10 \mathrm{ng} / \mathrm{ml}$, Roche Diagnositics, Indianapolis, IN) as described (25). $2 \times 10^{5}$ NSCs were suspended in culture medium containing b-FGF, chicken embryo extract, and $\mathrm{N}_{2}$ supplement and placed into the upper chamber of the transwell apparatus, with HB-EGF $(0-100 \mathrm{ng} / \mathrm{ml})$ added to the lower chamber. After $24 \mathrm{~h}$ of incubation, inserts were washed, fixed in $100 \%$ methanol, stained with $0.2 \%$ crystal violet, and cells trapped in the membrane insert pores were quantified using an inverted microscope (Carl Zeiss Microscopy LLC, Thornwood, NY). Each group had 12 slides, and three random visual fields in each slide were used for cell counting.

\section{Murine Model of Experimental NEC and Experimental Design}

The following experimental protocol followed the guidelines for the ethical treatment of experimental animals as approved by the Institutional Animal Care and Use Committee of the Research Institute at Nationwide Children's Hospital (protocol \#02205 AR). C57/BL6 mice were randomized into eight groups: (i) breastfed $(n=22)$; (ii) breastfed + i.p. delivery of NSC $(n=10)$; (iii) NEC ( $n=48)$; (iv) NEC + NSC i.p. (20,000 cells, single injection; $n=39$ ); (v) NEC + gastric gavage of HB-EGF ( $800 \mu \mathrm{g} / \mathrm{kg} / \mathrm{dose} ; n=26)$; (vi) NEC + HB-EGF + NSC i.p. $(n=31)$; (vii) NEC + scramble-transfected NSC i.p. $(n=43)$; and (viii) NEC + HB-EGF-overexpressing NSC i.p. $(n=27)$. NEC was induced using a modification of the model described by Barlow et al. (26) and modified for mice by Jilling et al. (27). Pups were collected after vaginal delivery and prior to breastfeeding. The pups were fed Similac 60/40 (Ross Pediatrics, Columbus, $\mathrm{OH}$ ) formula fortified with Esbilac powder (Pet-Ag, New Hampshire, IL) which provided $836.8 \mathrm{~kJ} / \mathrm{kg}$ per day. Starting at $0.03 \mathrm{ml}$ of formula every $3 \mathrm{~h}$ beginning $2 \mathrm{~h}$ after delivery, the volume of formula was advanced to a maximum of $0.05 \mathrm{ml}$ per feed by the fourth day of life. Pups were exposed to hypoxia ( $100 \%$ nitrogen $\times 1 \mathrm{~min})$ followed by hypothermia $\left(4{ }^{\circ} \mathrm{C} \times 10 \mathrm{~min}\right)$ every $12 \mathrm{~h}$. Pups were killed upon development of clinical signs of NEC including abdominal distention, bloody stools, or respiratory distress. Any surviving pups were killed $96 \mathrm{~h}$ after birth. Pups treated with enteral HB-EGF received $800 \mu \mathrm{g} / \mathrm{kg} /$ dose added to each feed. Pups receiving either HB-EGF-transfected or scramble-transfected NSC had 20,000 cells suspended in $30 \mu$ of Hanks' balanced Salt solution (HBSS) and administered via i.p. injection $2 \mathrm{~h}$ after birth. Pups not treated with NSC received an equal volume of HBSS. Control pups were breastfed normally. Intestines and blood samples were harvested immediately upon euthanasia.

\section{Histologic Injury Grading}

Mouse small intestines were fixed in $4 \%$ paraformaldehyde, paraffinembedded, and hematoxylin and eosin-stained sections obtained. Intestinal injury was measured using a standard histological scoring system in which injury was graded as follows: grade 0 , normal intestine; grade 1, epithelial cell lifting or separation; grade 2 , necrosis to the mid villus level; grade 3 , necrosis of the entire villus; and grade 4 , transmural necrosis. Any injury of grade 2 or above was considered consistent with NEC. Six segments of intestines were analyzed for each mouse. Twenty-four fields from each section were viewed and graded blindly by two independent investigators.

\section{Intestinal Permeability}

Intestinal permeability in mice subjected to experimental NEC was measured as the serum concentration of fluorescein isothiocyanantelabeled dextran molecules (FD70, molecular weight 73,000; SigmaAldrich). FD70 $(750 \mathrm{mg} / \mathrm{ml})$ was administered enterally $4 \mathrm{~h}$ prior to euthanasia and serum harvested. The serum concentration of fluorescein isothiocyanante-dextran was measured using a fluorescent plate reader with a $492 / 515 \mathrm{~nm}$ filter set.

\section{Immunohistochemistry}

Murine intestinal tissue sections $(4 \mu \mathrm{m}$ thickness) were double stained with anti-Ki67 (1:400; Abcam, Cambridge, MA) and antiEGFP (1:500; Thermo, Rockford, IL) antibodies or with anti-human HB-EGF $(5 \mu \mathrm{g} / \mathrm{ml}$; R\&D Systems, Minneapolis, MN) and anti-EGFP (1:500) antibodies or with anti-human neuronal protein $\mathrm{HuC} / \mathrm{HuD}$ (HuC/D; a pan neuronal cell marker; $5 \mu \mathrm{g} / \mathrm{ml}$; Life Technology, Eugene, OR) and anti-EGFP (1:500) antibodies, followed by speciesspecific secondary antibodies conjugated to $\mathrm{Cy} 3$ or fluorescein isothiocyanate, respectively. Coverslips were mounted onto glass slides with mounting medium containing 4',6-diamidino-2-phenylindole (DAPI) (Vector, Burlingame, CA). Images were visualized using a Zeiss LSM 700 confocal microscope (Zeiss) with a $\times 40$ objective. Five serial histologic sections per pup were examined.

\section{Intestinal Motility Assay}

The following experimental protocol followed the guidelines for the ethical treatment of experimental animals as approved by the Institutional Animal Care and Use Committee of the Research Institute at Nationwide Children's Hospital (protocol \#04203AR). Intestinal motility was quantified in rat pups exposed to a modification of the murine NEC model described above. Rat pups were exposed to NEC stress for $3 \mathrm{~d}$, and rat pups that survived received an i.p. injection of HB-EGF-transfected or scramble-transfected NSC (50,000 cells suspended in $50 \mu \mathrm{l}$ of HBSS). Nontreated pups received an equal volume of HBSS. Pups then received normal formula or normal formula supplemented with HB-EGF for an additional $4 \mathrm{~d}$ to simulate recovery from NEC. Pups were made nil per os for $4 \mathrm{~h}$ followed by administration of $0.2 \mathrm{ml}$ methylene blue-labeled $10 \%$ dextrose solution (Sigma-Aldrich) delivered by gastric gavage and were killed 5 min after introduction of the dye. The distance from the pylorus to the most distal point of migration of the blue dye was measured as intestinal transit. The total length of the small intestine was measured, and the ratio of dye migration distance/total intestinal length was calculated, and transit times were expressed as a percentage of the total intestinal length.

\section{Statistical Analyses}

All parametric results are presented as mean \pm SD. The numbers of engrafted NSC and the serum concentration of fluorescein isothiocyanante-dextran were compared using ANOVA. NSC viability and migration were analyzed using ANOVA, with Dunnett's post hoc analysis used when needed. Relative mRNA expression levels were compared between each type of siRNA and its own control using twosample $t$-test. The proportions of NEC incidents were compared using 
Pearson's Chi-square test or Fisher's exact test as appropriate. $P$ values of $<0.05$ were considered statistically significant.

\section{STATEMENT OF FINANCIAL SUPPORT}

This research was supported by National Institutes of Health (Bethesda, MD, USA) grants R01 GM61193 and R01 DK74611 (G.E.B.).

Disclosure: The authors declare no conflicts of interest to disclose.

\section{REFERENCES}

1. Fitzgibbons SC, Ching Y, Yu D, et al. Mortality of necrotizing enterocolitis expressed by birth weight categories. J Pediatr Surg 2009;44:1072-5; discussion 1075-6.

2. Furness JB. Types of neurons in the enteric nervous system. J Auton Nerv Syst 2000;81:87-96.

3. Boeckxstaens GE. Understanding and controlling the enteric nervous system. Best Pract Res Clin Gastroenterol 2002;16:1013-23.

4. Neunlist M, Van Landeghem L, Mahé MM, Derkinderen P, des Varannes SB, Rolli-Derkinderen M. The digestive neuronal-glial-epithelial unit: a new actor in gut health and disease. Nat Rev Gastroenterol Hepatol 2013;10:90-100.

5. Neu J. Gastrointestinal development and meeting the nutritional needs of premature infants. Am J Clin Nutr 2007;85:629S-34S.

6. Zhou Y, Yang J, Watkins DJ, et al. Enteric nervous system abnormalities are present in human necrotizing enterocolitis: potential neurotransplantation therapy. Stem Cell Res Ther 2013;4:157.

7. Almond S, Lindley RM, Kenny SE, Connell MG, Edgar DH. Characterisation and transplantation of enteric nervous system progenitor cells. Gut 2007;56:489-96.

8. Reekmans K, Praet J, Daans J, et al. Current challenges for the advancement of neural stem cell biology and transplantation research. Stem Cell Rev 2012;8:262-78.

9. Besner G, Higashiyama S, Klagsbrun M. Isolation and characterization of a macrophage-derived heparin-binding growth factor. Cell Regul 1990;1:811-9.

10. Higashiyama S, Abraham JA, Miller J, Fiddes JC, Klagsbrun M. A heparinbinding growth factor secreted by macrophage-like cells that is related to EGF. Science 1991;251:936-9.

11. Feng J, El-Assal ON, Besner GE. Heparin-binding epidermal growth factor-like growth factor decreases the incidence of necrotizing enterocolitis in neonatal rats. J Pediatr Surg 2006;41:144-9; discussion 9.

12. Feng J, Besner GE. Heparin-binding epidermal growth factor-like growth factor promotes enterocyte migration and proliferation in neonatal rats with necrotizing enterocolitis. J Pediatr Surg 2007;42:214-20.

13. Chen CL, Yu X, James IO, et al. Heparin-binding EGF-like growth factor protects intestinal stem cells from injury in a rat model of necrotizing enterocolitis. Lab Invest 2012;92:331-44.
14. Yang J, Watkins D, Chen CL, Bhushan B, Zhou Y, Besner GE. Heparinbinding epidermal growth factor-like growth factor and mesenchymal stem cells act synergistically to prevent experimental necrotizing enterocolitis. J Am Coll Surg 2012;215:534-45.

15. Watkins DJ, Yang J, Matthews MA, Besner GE. Synergistic effects of HB-EGF and mesenchymal stem cells in a murine model of intestinal ischemia/reperfusion injury. J Pediatr Surg 2013;48:1323-9.

16. Metzger M, Caldwell C, Barlow AJ, Burns AJ, Thapar N. Enteric nervous system stem cells derived from human gut mucosa for the treatment of aganglionic gut disorders. Gastroenterology 2009;136:2214-25.e1-3.

17. Hotta R, Stamp LA, Foong JP, et al. Transplanted progenitors generate functional enteric neurons in the postnatal colon. J Clin Invest 2013;123: 1182-91.

18. Ideguchi M, Shinoyama M, Gomi M, Hayashi H, Hashimoto N, Takahashi J. Immune or inflammatory response by the host brain suppresses neuronal differentiation of transplanted ES cell-derived neural precursor cells. J Neurosci Res 2008;86:1936-43.

19. Rocourt DV, Mehta VB, Besner GE. Heparin-binding EGF-like growth factor decreases inflammatory cytokine expression after intestinal ischemia/ reperfusion injury. J Surg Res 2007;139:269-73.

20. Yang J, Su Y, Zhou Y, Besner GE. Heparin-binding EGF-like growth factor (HB-EGF) therapy for intestinal injury: application and future prospects. Pathophysiology 2014;21:95-104.

21. Zhou Y, James I, Besner GE. Heparin-binding epidermal growth factorlike growth factor promotes murine enteric nervous system development and enteric neural crest cell migration. J Pediatr Surg 2012;47: 1865-73.

22. Nishi E, Prat A, Hospital V, Elenius K, Klagsbrun M. N-arginine dibasic convertase is a specific receptor for heparin-binding EGF-like growth factor that mediates cell migration. EMBO J 2001;20:3342-50.

23. Hoshino H, Uchida T, Otsuki T, et al. Cornichon-like protein facilitates secretion of HB-EGF and regulates proper development of cranial nerves. Mol Biol Cell 2007;18:1143-52.

24. Fumagalli P, Accarino M, Egeo A, et al. Human NRD convertase: a highly conserved metalloendopeptidase expressed at specific sites during development and in adult tissues. Genomics 1998;47:238-45.

25. Zhang C, Ge X, Lok K, Zhao L, Yin M, Wang ZJ. RhoC involved in the migration of neural stem/progenitor cells. Cell Mol Neurobiol 2014;34:409-17.

26. Barlow B, Santulli TV, Heird WC, Pitt J, Blanc WA, Schullinger JN. An experimental study of acute neonatal enterocolitis-the importance of breast milk. J Pediatr Surg 1974;9:587-95.

27. Jilling T, Simon D, Lu J, et al. The roles of bacteria and TLR4 in rat and murine models of necrotizing enterocolitis. J Immunol 2006;177: 3273-82. 